Provided for non-commercial research and education use. Not for reproduction, distribution or commercial use.

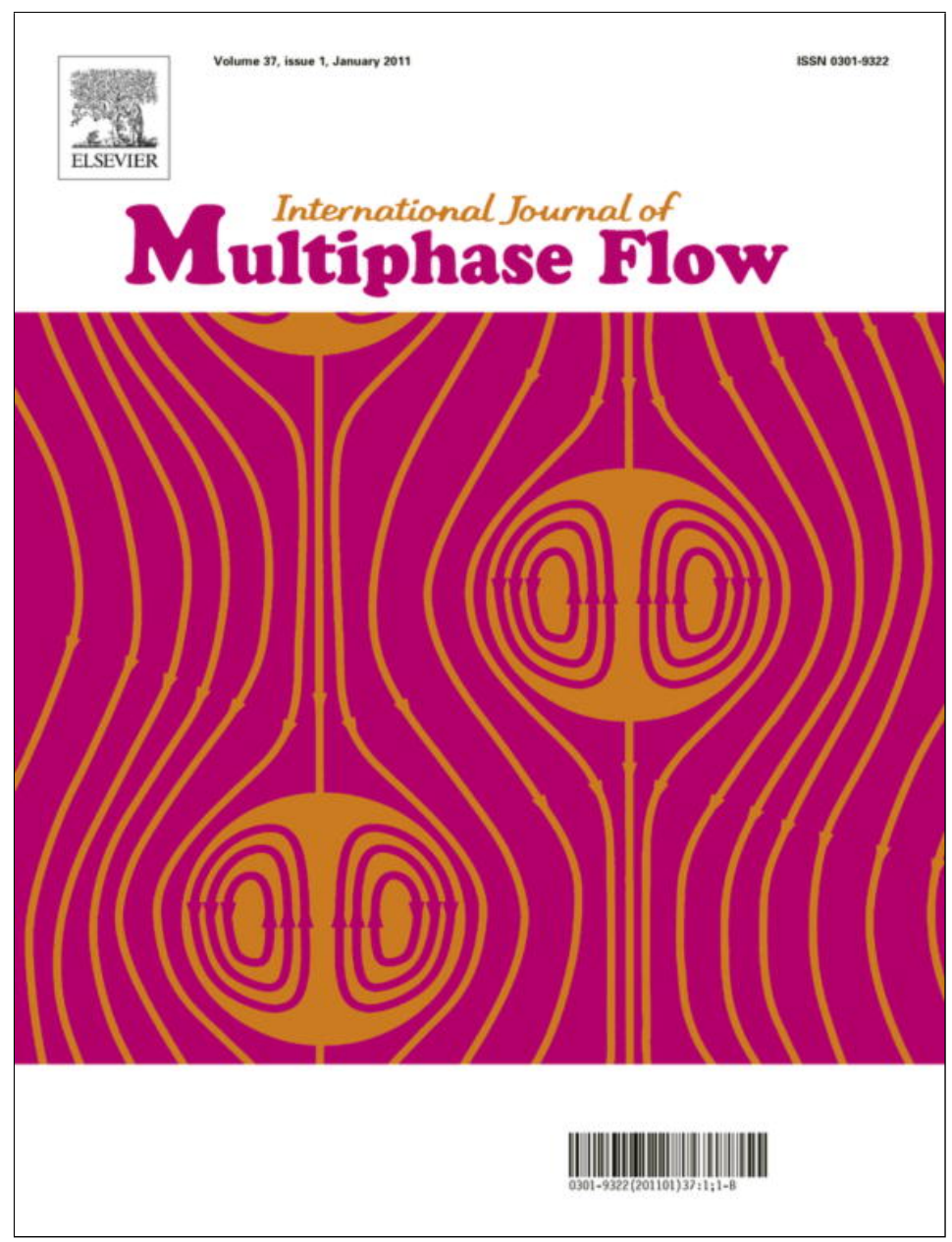

(This is a sample cover image for this issue. The actual cover is not yet available at this time.)

This article appeared in a journal published by Elsevier. The attached copy is furnished to the author for internal non-commercial research and education use, including for instruction at the authors institution and sharing with colleagues.

Other uses, including reproduction and distribution, or selling or licensing copies, or posting to personal, institutional or third party websites are prohibited.

In most cases authors are permitted to post their version of the article (e.g. in Word or Tex form) to their personal website or institutional repository. Authors requiring further information regarding Elsevier's archiving and manuscript policies are encouraged to visit:

http://www.elsevier.com/copyright 


\title{
Laboratory experiments on DNAPL gravity fingering in water-saturated porous media
}

\author{
Khalifa Nsir ${ }^{a}$, Gerhard Schäfer ${ }^{\mathrm{a}, *}$, Raphaël di Chiara Roupert ${ }^{\mathrm{a}}$, Olivier Razakarisoa ${ }^{\mathrm{a}}$, Renaud Toussaint ${ }^{\mathrm{b}}$ \\ a Laboratoire d'Hydrologie et de Géochimie de Strasbourg (LHyGeS), UMR 7517, Université de Strasbourg/EOST, Centre National de la Recherche Scientifique (CNRS), \\ 1 rue Blessig, 67084 Strasbourg, France \\ ${ }^{\mathrm{b}}$ Institut de Physique du Globe (IPGS), UMR 7516, Université de Strasbourg/EOST, CNRS, 5 rue René Descartes, 67084 Strasbourg, France
}

\section{A R T I C L E I N F O}

\section{Article history:}

Received 15 March 2011

Received in revised form 6 December 2011

Accepted 8 December 2011

Available online 16 December 2011

\section{Keywords:}

Gravity-driven instabilities

Immiscible displacement

Porous medium

DNAPL

Optical fibers

Residual saturation

\begin{abstract}
A B S T R A C T
Laboratory experiments were carried out at the Darcy scale to investigate the gravity-driven fingering phenomenon of immiscible two-phase flow of water and a dense nonaqueous-phase liquid (DNAPL) such as trichloroethylene (TCE). Rate-controlled displacement experiments were performed on a homogenous sand-filled column under various displacement conditions. Several system parameters (e.g. flow rate, flow mode (upward flow, downward flow) and mean grain-size diameter of the porous medium) were varied in the experimental programme. Optical fiber sensors were developed to quantify the spatial distribution of the advancing displacement front in a given control section of the experimental device. Following each experiment, multi-point measurements of the remaining TCE saturation were obtained by in situ soil sampling. The resulting DNAPL distribution was heterogeneous even though the medium was homogeneous sand. Higher DNAPL injection rates and lower medium permeability both reduced gravity fingering. This is because viscous forces stabilize the advancing front with pressure gradients increasing as function of the injection rate and decreasing as function of the permeability. Average residual TCE saturations obtained by mass-balance in the experiment after a complete drainage-imbibition cycle were influenced by the mean grain-size diameter of the porous medium but were not affected by the flow mode of the primary drainage process.
\end{abstract}

(c) 2011 Elsevier Ltd. All rights reserved.

\section{Introduction}

Characterization and quantification of the migration of immiscible liquids in ground-water are topics that have received considerable attention in recent years. Recent studies of two-phase immiscible flows have been motivated by the need to treat spills and leaks of so-called dense nonaqueous-phase liquids (DNAPLs), such as trichloroethylene (TCE), which can severely impact the quality of subsurface water supplies (Kueper and Frind, 1989; Birovljev et al., 1991; Fayers and Zhou, 1996; Bettahar et al., 1999; Benremita and Schäfer, 2003; Bohy et al., 2004). Unstable immiscible displacement can form viscous, capillary, and/or gravity fingers. These fingers propagate rapidly, causing early breakthrough relative to stable displacement (Brailovsky et al., 2006).

The current physical understanding of fingering processes has been developed primarily through linear stability analysis (Yao and Hendrickx, 2001). While this theory provides information about the displacement process under unstable conditions and the practical growth rate of fingers for small amplitude perturbations, it neither predicts nor explains many significant features

\footnotetext{
* Corresponding author.

E-mail address: schafer@unistra.fr (G. Schäfer).
}

observed in laboratory experiments. The assumptions inherent to the linear analysis are somewhat limiting with respect to actual field conditions. Fingering processes were initially studied in 2D experiments, such as empty Hele-Shaw cells, as in the fundamental work of Saffman and Taylor (1958). These cells are composed of two closely spaced parallel plates, and the motion of fluids in the system is mathematically analogous to that of two-dimensional flow in a porous medium. Later on, porous media was added between the plates of Hele-Shaw cells for better reproduction of the random character of porous geometry. Observations from laboratory experiments on instabilities have allowed researchers to both test the predictions of the linear stability theory for fingers and to discover a number of unexpected features of fingering flow fields, in which instabilities arise from the presence of gravity (Frette et al., 1997), viscosity (Løvoll et al., 2004; Toussaint et al., 2005; Løvoll et al., 2011), both gravity and viscosity (Méheust et al., 2002; Nicholl and Glass, 2005) or capillarity when the displacement is very slow (Lenormand and Zarcone, 1989; Ferer et al., 2007; Christophe et al., 2010).

Laboratory studies have also been conducted on soil columns to characterize the behavior of DNAPL displacement in saturated porous media and to better understand the complexities of DNAPL migration in environmental media (Khataniar and Peters, 1992; Riaz and Tchelepi, 2006; Tullis and Wright, 2007; Schwille, 1988) 
conducted large column experiments $(1 \mathrm{~m}$ high, $0.4 \mathrm{~m}$ diameter) with perchloroethylene (PCE) in a low-permeability saturated sand $\left(K=2 \times 10^{-4} \mathrm{~m} \mathrm{~s}^{-1}\right)$. He found that the PCE front was not uniform or even bulb-shaped. Instead, the PCE penetrated as tendrils or fingers in the interior of the sand body. Schwille (1988) also investigated PCE penetration into water-saturated glass beads with diameters ranging from 0.49 to $0.7 \mathrm{~mm}$. The DNAPL was observed to penetrate into the saturated zone in a finger-like stream. Anderson et al. (1992) described the common occurrence of DNAPLs in the saturated zone of aquifers in the form of fingered plumes and pools that cause erratic contaminant distributions. Glass and Nicholl (1996) reported that a complicated pattern of interacting fingers resulted from their stratified soil experiments; and this limited the number of isolated fingers for which velocity and diameter data could be collected. To explore the wetting phase saturation levels in fingers, Geiger and Durnford (2000) and Dicarlo (2004) conducted one-dimensional fingering experiments using small cylindrical columns in which they examined the variation in DNAPL saturation along the finger length. Their results suggested that the propagating fingertip is near full saturation, and the saturation level decreases in the direction of the finger tail. Glass et al. (2000) used a high-resolution light transmission method to investigate gravity-destabilized non-wetting phase invasion $\left(\mathrm{CO}_{2}\right.$ and trichloroethylene) in a macro-heterogeneous porous medium. They observed a series of fingers and pools, behind the growing front, as a sequence of gravity-stabilized and gravity-destabilized displacements within the heterogeneous medium.

To describe the two-phase liquid-liquid displacement in a porous medium, various dimensionless parameters such as the capillary number $N_{\mathrm{c}}$ (ratio of viscous to capillary forces), the viscosity ratio $M$ (ratio between the displaced fluid viscosity and the displacing fluid viscosity), and the Bond number $N_{\mathrm{b}}$ (ratio of gravity to capillary forces) are commonly used in the literature (Gioia and Urciuolo, 2006). These numbers are defined as follows:

$N_{\mathrm{c}}=\frac{v \mu_{w}}{\sigma_{\mathrm{ow}} \cos \theta}$

where $v$ is the Darcy velocity $\left(\mathrm{L} \mathrm{T}^{-1}\right), \mu_{\mathrm{w}}\left(\mathrm{M} \mathrm{L}^{-1} \mathrm{~T}^{-1}\right)$ is the dynamic viscosity of the wetting fluid, $\sigma_{\mathrm{ow}}\left(\mathrm{M} \mathrm{T}^{-2}\right)$ is the interfacial tension between the non-wetting (oil) and wetting fluid (water) and $\theta(-)$ is the contact angle at the solid-water-DNAPL interface;

$M=\frac{\mu_{1}}{\mu_{2}}$

where $\mu_{1}\left(\mathrm{M} \mathrm{L}^{-1} \mathrm{~T}^{-1}\right)$ is the dynamic viscosity of the displacing fluid and $\mu_{2}\left(\mathrm{M} \mathrm{L}^{-1} \mathrm{~T}^{-1}\right)$ is the dynamic viscosity of the displaced fluid, and

$N_{\mathrm{b}}=\frac{\Delta \rho g a^{2} \cos (\alpha+\pi)}{\sigma_{\mathrm{ow}} \cos \theta}$

where $\Delta \rho\left(\mathrm{M} \mathrm{L}^{-3}\right)$ is the difference between the density of displacing fluid and the density of displaced fluid, $g\left(\mathrm{~L} \mathrm{~T}^{-2}\right)$ is gravitational acceleration, $a(\mathrm{~L})$ is the mean grain diameter of the porous medium, and $\alpha$ $(-)$ is the angle formed by the flow vector and gravity vector. When gravity forces are involved, the Bond number can be either positive or negative, depending on the value of $\alpha$. The common convention is that $N_{\mathrm{b}}$ is considered positive when flow is gravity-stabilized, whereas $N_{\mathrm{b}}$ is considered negative when the gravity acting to destabilize the displacement. In the context of vertical gravity-driven DNAPL migration in porous media, $N_{\mathrm{b}}$ is usually negative.

When two liquids are in contact in a porous medium, the denser fluid tends to sink to the bottom, and the upward force that this heavier fluid exerts on the lighter fluid to balance its weight is known as the buoyancy force. In the absence of buoyancy forces, the fluid patterns formed by immiscible displacement of a wetting fluid by a non-wetting fluid can be divided into three regimes (stable, viscous fingering, and capillary fingering regimes) separated by an intermediate transition zone. Lenormand et al. (1983) developed a phase diagram used to identify the location of the different regimes, and thus of the transitional region between them, in an $M$, $N_{\mathrm{c}}$ space. Ewing and Berkowitz (1998) noted that the phase diagram relating viscous, capillary, and stable flow regimes should contain a third dimension to account for gravity and buoyancy forces, given by the Bond number $N_{\mathrm{b}}$.

The quantitative parameters previously described have also been applied to estimate the mobility of DNAPLs in porous media in terms of temporal variation of DNAPL saturation profiles during immiscible displacements (Pennell et al., 1996; Helen and Paul, 1997; Hunt et al., 1998; Theodoropoulou et al., 2005; Glen et al., 2006). Several methods have been used to study fluid saturations during the immiscible fluid displacement process. In the past resistivity measurements (Leverett, 1939) and microwave absorption (Parsons and Marathon, 1975) provided area-average fluid saturations. More recently, techniques such as radioisotope tracing/ Gamma cameras (Zhang and Smith, 2001), X-ray tomography methods (Glass et al., 2000) were able to deliver two-dimensional projections of the fluid saturation distribution and provide realtime visualization of the advancing front. In these cases, the ability to reproduce physical measurements was excellent. However, if excellent images were obtained and the exact macroscopic structure of fingers has to be provided, the application of this method was limited to either 2D flow cells, or fairly small 3D flow. One general conclusion from water-flooding investigations is that DNAPL saturation in porous media strongly depends on the interfacial tension between the immiscible liquids, the wettability of the solids with respect to the immiscible liquids, and the pore water velocity attained during water flooding (Nguyen et al., 2006).

The aforementioned methods have not provided enough accurate data for a conclusive study of displacement instability processes. In addition, few experimental studies on immiscible displacement have considered buoyancy forces, and relevant data from experiments depicting the development of DNAPL gravity fingering are thus needed. Additionally, there is still no clear experimental evidence on the effect of flow rates, mode flow (gravity-stabilized, gravity-destabilized), and texture of the porous medium on the occurrence of this phenomenon. A consistent issue is that natural porous media are opaque, and we need to look at the pore scale to understand to understand how the fingers evolve.

The overall aim of the present work is to quantify displacement instabilities in a saturated homogeneous porous medium. The experimental approach is original as it is based on the use of optical fibers to quantify locally the arrival times of the DNAPL/water front at different points within a control section of the porous medium. The column experiments comprise a series of drainage experiments with TCE as the non-wetting fluid and water as the wetting fluid. A second set of experiments focus on a complete drainageimbibition cycle to remobilize the TCE entrapped during the primary drainage in the porous medium. Various displacement configurations were considered for study of the influence of specific model parameters on the displacement process, such as the direction of flow, the velocity of the displacing fluid, and the texture of the porous medium. The experiments were analyzed with respect to both distribution of water/DNAPL front velocities, and the DNAPL saturation in the column. In addition, conclusions about displacement instability were then drawn.

\section{Materials and methods}

\subsection{Experimental setup}

Laboratory experiments were conducted on a transparent glass column with a total length of $68 \mathrm{~cm}$ and an inner diameter of 
$10 \mathrm{~cm}$. Two porous media were used: medium sand and fine sand. The characteristic properties of both porous media, such as intrinsic permeability $(k)$, mean grain size diameter $\left(d_{50}\right)$, uniformity coefficient $(U)$, and porosity $(\eta)$ were determined experimentally (Table 1).

Trichloroethylene (TCE) was chosen as the DNAPL for the experiments because it is among the most frequently detected contaminants in subsurface environments. At $20^{\circ} \mathrm{C}$, TCE has a density of $1.463 \mathrm{~g} / \mathrm{cm}^{3}$, a viscosity of $0.0056 \mathrm{~g} / \mathrm{s} / \mathrm{cm}$, and a solubility in water of $1300 \mathrm{mg} / \mathrm{L}$ (Jellali et al., 2003). In the column experiments, a dye (Organol Red BS) was added to TCE to allow visual detection of the DNAPL during the experiments; the dye concentration was approximately $5 \mathrm{mg} / \mathrm{L}$. Organol Red BS is highly soluble in TCE, and does not modify its chemical and physical properties in the range of concentrations applied (Jellali et al., 2001). A high-accuracy peristaltic pump with Teflon tubing and interchangeable heads for flow rates up to $200 \mathrm{~mL} / \mathrm{min}$ was used to inject the displacing fluid in the sand-filled column. A 2-cm layer of coarse sand was placed at the top and bottom of the sand column to ensure uniform flow conditions in the inlet and outlet sections of the studied sand column. Pressures at the inlet and outlet were measured using a pressure transducer (Cerabar T-PMC131). Eight optical fiber sensors were developed (see Section 2.2) to measure the arrival time of the DNAPL front at different points within a section located halfway up the column. The measurement recordings were acquired using an Agilent 34970A Data Acquisition device connected to a personal computer. The experimental setup used for running the displacement experiments is illustrated in Fig. 1.

The experimental setup was designed to produce a controlled and well-defined injection of TCE in an initially water-saturated homogeneous porous medium. Filling of the column was carried out with dry sand added regularly and successively in layers of several centimeters to a $10-\mathrm{cm}$ deep water layer at the bottom of the sand column, which was then mixed from bottom to top with a thin rigid rod. Next, the sides of the glass column were gently tapped to encourage the sand to settle tightly. This method was previously demonstrated by Nsir (2009) to yield packed columns with highly reproducible and uniform physical properties. To ensure that the sand filling had the same perfect water-wet characteristics in each run, the glass column was refilled with fresh sand for each experiment. Before the start of each displacement experiment, the column was flushed with de-aired water to ensure complete water saturation of the sand, and to measure the hydraulic conductivity of the sand column. Once steady water flow was achieved, TCE was injected through the injection port of the packed column at a constant flow rate. The outlet section of the column was kept at a constant water pressure. The optical fiber sensors might disturb locally the flow field but the effect of their emplacement has no major impact on the conclusions drawn about displacement instability as their emplacements in the porous medium were not changed during the different studied displacement scenarios.

Two displacement modes were studied in the experiments, referred to as vertical-upward and vertical-downward displacement later on in the study. In the first set of studies, drainage experiments were run in which TCE was the non-wetting fluid and water

Table 1

Properties of the porous media used in the experiments.

\begin{tabular}{lllll}
\hline & $\begin{array}{l}\text { Intrinsic } \\
\text { permeability } \\
K\left(\mathrm{~m}^{2}\right)\end{array}$ & $\begin{array}{l}\text { Mean grain } \\
\text { diameter } d_{50} \\
(\mathrm{~mm})\end{array}$ & $\begin{array}{l}\text { Uniformity } \\
\text { coefficient } \\
U(-)\end{array}$ & $\begin{array}{l}\text { Porosity } \\
(\eta)\end{array}$ \\
\hline Medium sand & $9 \times 10^{-11}$ & 0.43 & 2.1 & 0.43 \\
Fine sand & $5 \times 10^{-12}$ & 0.17 & 2.3 & 0.40 \\
\hline
\end{tabular}

the wetting fluid. Here, the displacement is generally unstable according to the contrast of density and the unfavorable viscosity ratio $M(M=0.58<1)$ that existed between the immiscible fluids. The second set of experiments is primary drainage followed by water flooding to mobilize the TCE entrapped in the porous medium. The system constitutes a complete drainage-imbibition cycle. To reach residual TCE saturations, a large range of water flow rates was used. In practice, the critical value of the capillary number at which oil recovery becomes significant has been found to be in the range of between $10^{-5}$ and $10^{-4}$. As the capillary number in our experiments is typically in the order of $10^{-6}$ for ordinary water flooding, flow rate varied in the laboratory experiment from 40 up to $170 \mathrm{~mL} / \mathrm{min}$, corresponding to a capillary number $N_{\mathrm{c}}$ of $4.1 \times 10^{-6}$ to $16.8 \times 10^{-6}$. Residual TCE saturation after imbibition (water-flooding) was examined under a range of experimental conditions, including direction of flow (upward or downward), displacing fluid velocity, and the mean pore size (medium or fine sand). Tables 2 and 3 summarize the different parameters varied in drainage experiments and experiments conducted to study drainage-imbibition cycle, respectively.

A major issue in the experimental studies was how to quantify the preferential pathways of DNAPL during its migration in a saturated porous medium. The fingering process was recorded using specially designed optical fiber sensors to measure the arrival time of the water/DNAPL front at eight points in a control section located halfway up the column. To obtain visual information on the fingering events that appeared in the sand column, digital images were taken of the red-colored DNAPL blobs observed at the glass wall of the column. For each displacement experiment, the DNAPL breakthrough at the column outlet was determined by measuring the quantity of TCE leaving the column. The extent of local DNAPL saturation was determined by collecting and analyzing sand cores after each experiment (see Section 2.3).

\subsection{Optical fiber sensors}

The experiment used optical fibers to quantify the DNAPL migration pathways through the saturated sand. This technique, widely used in industrial applications, can act as an active sensing mechanism and is generally referred to as an intrinsic optical fiber sensor. Similar sensors have found application in chemical, biochemical, biomedical, and environmental sensing (Sharma and Guptal, 2006; Gupta and Verma, 2009). The general structure of the developed optical fiber sensor is shown in Fig. 2. It consists of an LED as the optical source, an optical fiber that transfers the optical signal, an optical detector (a photodiode), and processing electronics.

The sensing principle is based on the change of optical intensity modulation along the optical fiber. The tool converts the presence of a DNAPL into modified optical characteristics of the emitted signal. The original prototype version of the optical fiber sensor was conceived by VEGAS (Versuchseinrichtung für Grundwasser- und Altlastensanierung) of the University of Stuttgart (Barczewski and Marschall, 1992). As part of the PhD thesis of Nsir (2009), further improvements were undertaken to obtain a robust and sensitive tool for detecting the presence of DNAPL in porous media.

An optical fiber consists of a central core (through which light is guided) embedded in an outer cladding with a slightly lower refractive index. They are covered by a plastic jacket to mechanically protect the fiber and exclude stray light. Light transmission through an optical fiber occurs due to internal reflection at the core/cladding interface. Any change in reflection can be detected by a change in transmission. Because reflection depends on the refractive index of the medium surrounding the fiber, removing a portion of the cladding creates a zone that can detect DNAPL, due to the different refractive indices of water $\left(n_{\mathrm{w}}=1.33\right)$ and 


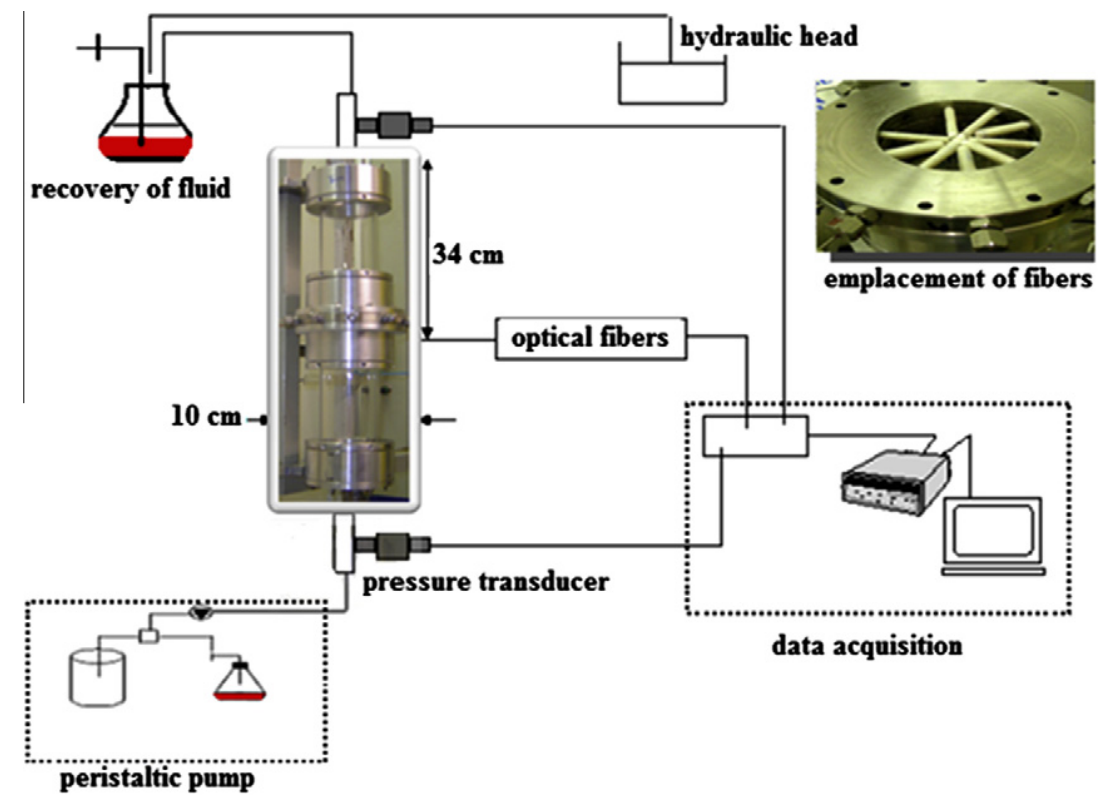

Fig. 1. Schematic representation of the experimental setup.

Table 2

Characteristics of drainage experiments.

\begin{tabular}{llllr}
\hline & Porous medium & Flow mode & $N_{\mathrm{c}}$ & $N_{b}$ \\
\hline Experiment 1 & Medium sand & Upward & $4.1 \times 10^{-6}$ & 0.58 \\
Experiment 2 & Medium sand & Downward & $4.1 \times 10^{-6}$ & 0.58 \\
Experiment 3 & Medium sand & Downward & $7.5 \times 10^{-6}$ & $-2.7 \times 10^{-5}$ \\
Experiment 4 & Fine sand & Downward & $4.1 \times 10^{-6}$ & 0.58 \\
\hline
\end{tabular}

Table 3

Characteristics of drainage-imbibition cycle experiments.

\begin{tabular}{lllll}
\hline & Porous medium & Drainage flow mode & Displacement condition & Imbibition flow mode \\
\hline Experiment 1 & Medium sand & Upward & Stabilizing gravity effect & Downward \\
Experiment 2 & Medium sand & Upward & Stabilizing gravity effect & Upward \\
Experiment 3 & Medium sand & Downward & Destabilizing gravity effect & Downward \\
Experiment 4 & Medium sand & Downward & Destabilizing gravity effect & Upward \\
Experiment 5 & Fine sand & Downward & Destabilizing gravity effect & Downward \\
\hline
\end{tabular}

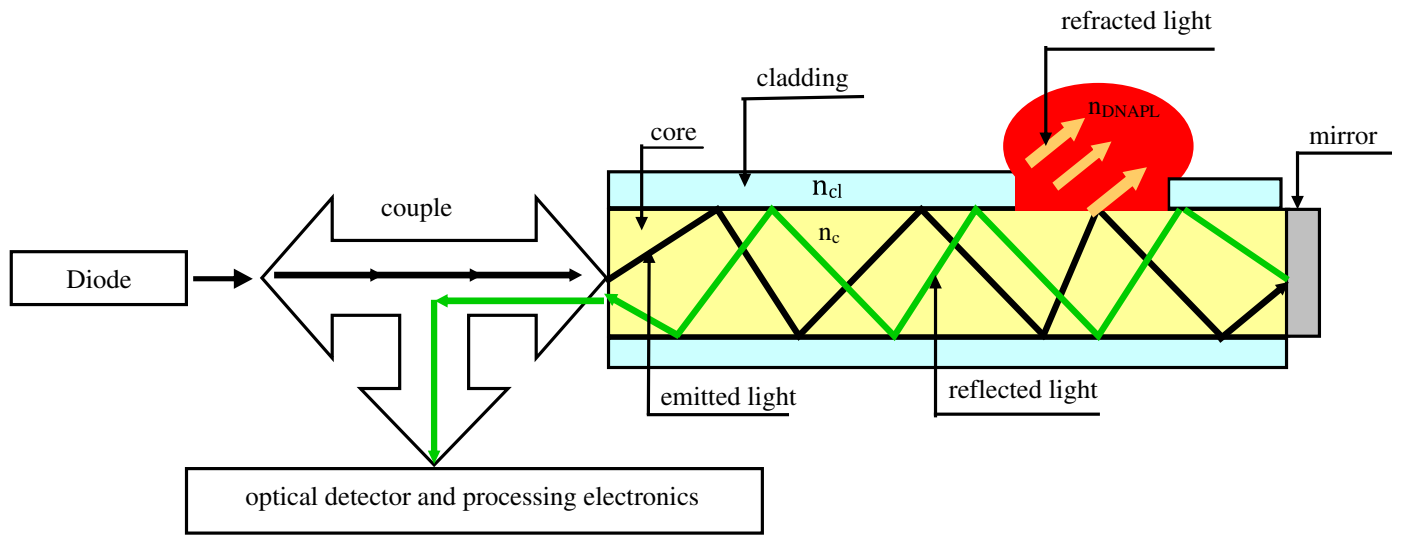

Fig. 2. Principle of the developed optical fiber sensor. $n_{\mathrm{cl}}$ and $n_{\mathrm{c}}$ are the refractive indices of the cladding and core of the optical fiber, respectively.

$\operatorname{DNAPL}\left(n_{\mathrm{DNAPL}}=1.46\right)$. Therefore, light intensity modulation can be brought about by refraction of rays in the DNAPL surrounding the modified cladding region.
The experimental work uses an all-silica multimode fiber with core/cladding/jacket external diameters of $600 / 675 / 690 \mu \mathrm{m}$. The optical fibers were approximately $50 \mathrm{~cm}$ long. The sensing element 
was prepared by decladding a small portion of the optical fiber (about $1 \mathrm{~cm}$ in length), which was then cleaved at its end face. Next, the polished surface of the end face was coated with a thin aluminum layer to create a mirror, using monomer vapor phase deposition (Nsir, 2009). The in situ deposition of the chemically active metal (aluminum) on the polished surface of fiber is achieved by suspending it in the monomer vapor phase deposition. The output face of the fiber is placed in front of a crucible filled with boil-off and heated to above $1000^{\circ} \mathrm{C}$. Evaporation of aluminum was carried out under a pressure of about $10^{-7} \mathrm{~atm}$. The coating and adhesion of the metal to the fiber surface were of high quality.

The developed sensors do not indicate of what percent of the proportion of DNAPL was in contact with the sensitive part of the fiber. They react for primary droplets of DNAPL being in contact with the sensitive part of the fiber. Therefore, detection of DNAPL in any sensor emplacement does not mean that surface surrounding of the sensing element is completely impregnated by DNAPL. An estimate of DNAPL saturation is thus not possible with the actual fiber tool.

\subsection{Quantification of DNAPL saturation}

For each drainage experiment, a total volume of TCE corresponding to approximately $70 \%$ of the pore volume of the packed sand was injected into the column at a constant flow rate of $40 \mathrm{~mL} / \mathrm{min}$. The amount of TCE entrapped in the porous medium was determined by mass balance of DNAPL inflow and outflow.

Residual saturations of TCE were established in the water-saturated sand column after a complete drainage-imbibition cycle. First, TCE was injected into the saturated porous medium, as in the drainage experiment. The flow direction was then reversed, and water was pumped through the column to displace the mobile TCE phase. To minimize the removal of entrapped TCE by dissolution the water injected into the sand-filled column did not exceed three times the total pore volume. If no TCE mobilization was observed after flushing with the chosen flow rate, an increased flow rate was applied to the sand column. For each flow rate, the DNAPL recovered in the effluent was collected in glass vials, and the volume of TCE displaced as free product measured. The average TCE saturation in the column was calculated per difference.

At the end of each displacement experiment (drainage experiment/complete drainage-imbibition cycle) the sand was sampled to obtain vertical profiles of local (entrapped/residual) TCE saturations in the saturated porous medium. Because of the emplacement of fibers, the sampling was achieved only above the sensor control section of the column. Local TCE saturations at various lateral positions and at different depths of the sand column were quantified using a soil sampler that probed a volume of roughly $2 \mathrm{~cm}^{3}$ (Nsir, 2009). The sampling grid used for this process was very fine $(5 \mathrm{~cm}$ spacing) in the vertical direction. At each sampling depth, one sample was taken on the axis of the column and two others on opposite points of the edges of the column section. TCE was extracted from these samples with methanol, and was measured by GC/FID.

\section{Results and discussion}

\subsection{Arrival time of the water/DNAPL front for a low injection rate}

To determine whether displacement instabilities occur, the arrival times of the DNAPL front were recorded at different points of the control section $(z=-34 \mathrm{~cm})$ during a drainage experiment conducted at a low injection rate of $40 \mathrm{~mL} / \mathrm{min}$, for both vertical-upward and vertical-downward flow modes. A normal distribution was fit to the arrival times, and normalized by the mean arrival time (Fig. 3). All sensors placed in the control section of the column detected
DNAPL for both gravity-destabilized and gravity-stabilized flow. This may be explained by the fact that only a small portion of the sensitive part of fiber may have been in contact with DNAPL droplets. In the case of the downward flow mode, the arrival times of the DNAPL/ water front varied widely, and their distribution is non-uniform. A standard deviation of nearly $70 \mathrm{~s}$ was obtained, whereas in the case of upward flow mode a standard deviation of only $9 \mathrm{~s}$ was calculated. This large difference is principally caused by density-driven fingering that develops in the case of the vertical-downward displacement. The low injection rate makes viscous forces negligible, so the capillary and buoyancy forces together dictate the movement of the advancing fluid. Given the contrast of both density and viscosity between the two fluids, the injected non-wetting fluid (TCE) prefers to move along preferential paths with low capillary resistances, thereby forming pronounced fingers. Capillary fingers, if formed, would be converted into gravity fingers as they elongated. A vertically oriented macroscopic finger may result that is composed of a sequence of larger cluster of entrapped TCE. In the case of verticalupward displacement, gravity plays a stabilizing role in the migration of DNAPL and compensates for the destabilizing viscosity ratio $M$ that exists between the two fluids. Under this condition, the water/TCE interface moves as a piston-like flow, and as a result, the recorded arrival times are very close together. This is consistent with the digital images taken of the red-colored TCE blobs observed at the glass wall of the column (Fig. 4). In the case of downward flow, TCE front was observed as small droplets or globules forming fingers and numerous protuberances, as described by Zhang and Smith (2001). In the case of upward flow, the TCE front followed a compact path as a succession of homogeneous layers alternating with thin discontinuities. This layering is an artifact of the packing method employed in the experiments. Here, $N_{\mathrm{b}}$ becomes positive in the system and its value of $2.7 \times 10^{-5}$ seems to be sufficient to generate a stable displacement regime independently of the values of both capillary number $\left(N_{\mathrm{c}}=4.1 \times 10^{-6}\right)$ and unfavorable viscosity ratio $(M=0.58)$.

In case of downward displacement, buoyancy forces speed up the downward movement of the TCE on account of its higher density as compared to water (Fig. 4b). Thus, the displacing fluid moves much faster than in the vertical-upward case, and a premature breakthrough time is established. This behavior was experimentally confirmed, with TCE breaking through at the outlet section of the column at nearly $30 \mathrm{~min}$ and $22 \mathrm{~min}$ for upward displacement and downward displacement, respectively.

\subsection{Average DNAPL saturation}

The average TCE saturations calculated by mass balance over the entire column are quite different for upward and downward

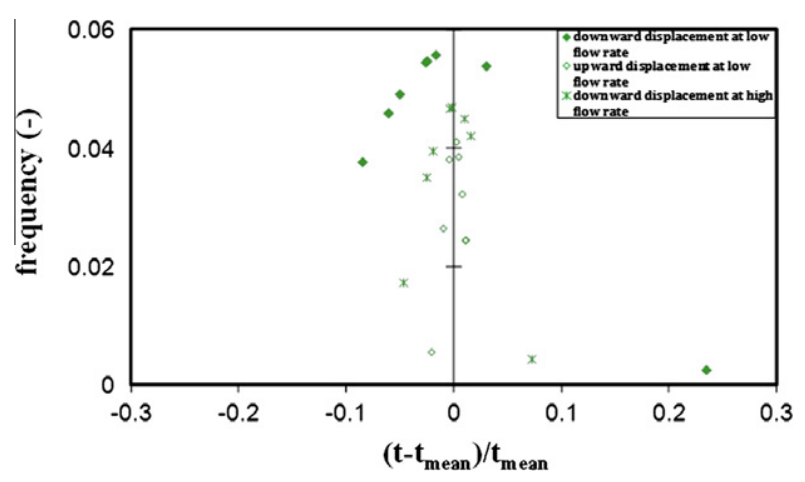

Fig. 3. Measured dimensionless arrival times of DNAPL front in both verticalupward flow mode and downward flow mode at low DNAPL injection rate and in downward flow mode at high DNAPL injection rate $\left(t_{\text {mean }}\right.$ is the average arrival time of DNAPL front): case of drainage of water-saturated medium sand. 
(a)

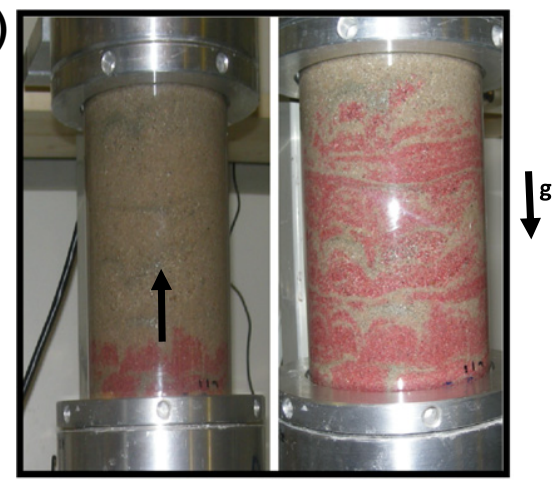

(b)

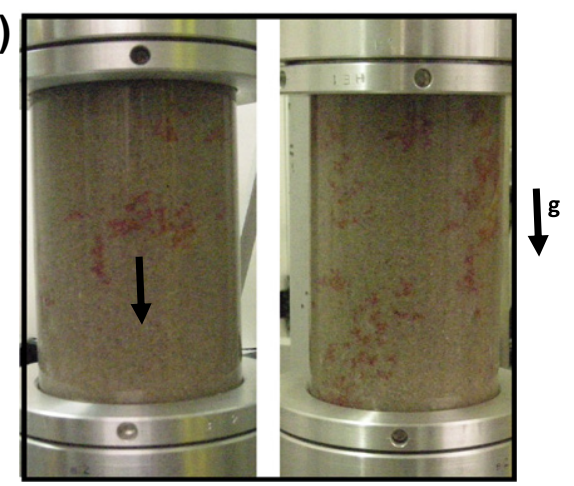

Fig. 4. TCE front observed on the column wall at the beginning ( $5 \mathrm{~min}$ ) and the end of experiment: (a) case of upward drainage of water-saturated medium sand at low DNAPL injection flow rate; (b) case of downward drainage of water-saturated medium sand at low DNAPL injection flow rate.

displacements (Fig. 5), and these observations confirm that density contrast plays a significant role in the migration of a DNAPL in a porous medium, particularly at low DNAPL injection rates. In the experiments, we determined an average TCE saturation of about $66 \%$ in the case of upward displacement, but only $33 \%$ for downward displacement. Furthermore, soil samples taken at the end of each drainage experiment clearly indicate that the TCE saturation front behavior is abrupt in the case of upward water displacement varying from $20 \%$ to $70 \%$ at a depth between 10 and $30 \mathrm{~cm}$. In the case of downward displacement, the TCE saturations are more or less uniformly distributed over the sampling depths and are characterized by a low saturation of about $16 \%$ (see Fig. 5 ). This difference is mainly due to the stable growth during upward displacement, whereas downward displacement is characterized by flow instabilities. The interactions between the capillary, buoyancy, and viscous forces result in a complex flow pattern characterized by ramified features as observed in Fig. $4 \mathrm{~b}$.

\subsection{DNAPL pressure at the inlet section}

Although the DNAPL saturation describes the distribution of displacement fluids in the porous medium, it is the pressure build-up that significantly determines the displacement of one fluid by another. Thus, to better understand the interplay between global pressure behavior and displacement condition (stable or unstable), the inlet TCE pressure was measured in the laboratory experiment for upward and downward displacement. Fig. 6a and b summarizes the TCE pressure measured as a function of time at the inlet section of the soil column for the cases of upward displacement and downward displacement, respectively. The boundary pressure applied to the outlet section of the experimental system corresponded to a water height of $18 \mathrm{~cm}$ and $86 \mathrm{~cm}$, respectively. As shown in Fig. 6a, the pressure at the inlet section

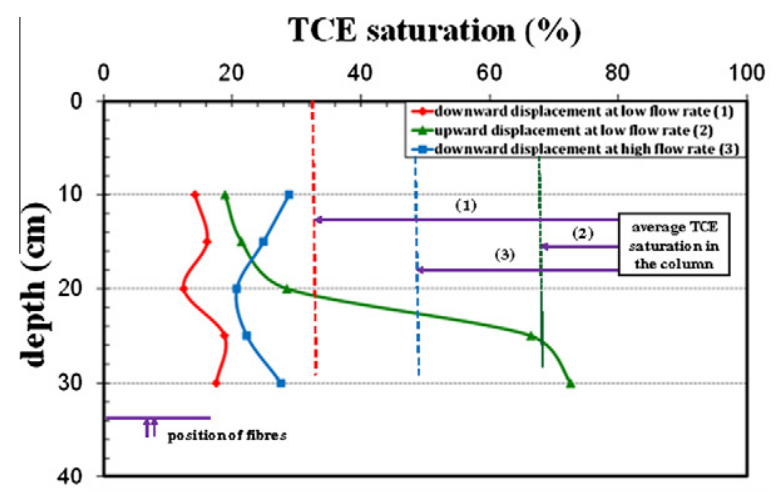

Fig. 5. Measured TCE saturation as function of depth and average TCE saturation in the column at the end of experiment for both vertical-upward and verticaldownward drainage of water-saturated medium at low DNAPL injection flow rate and vertical-downward drainage of water-saturated medium sand at high DNAPL injection flow rate.
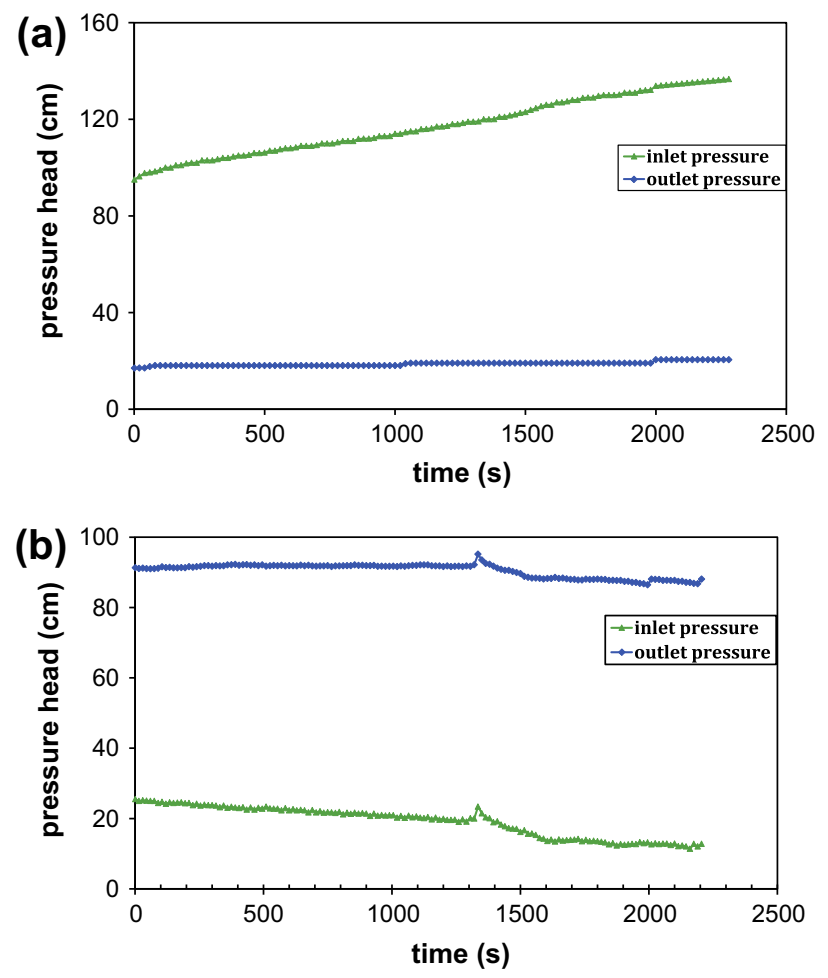

Fig. 6. Measured inlet and outlet pressure head as functions of time: (a) case of vertical-upward drainage of water-saturated medium sand at low DNAPL injection flow rate; and (b) case of vertical-downward drainage of water-saturated medium sand at low DNAPL injection flow rate.

increased with time in the case of vertical upward displacement. Thus, the water/DNAPL interface is stabilized by the gravity effect in this case. This confirms the findings of Løvoll et al. (2005). The potentially destabilizing influence of the viscosity ratio was not significant. The pressure increase is therefore caused by the high density of the invading fluid, which overrides the decrease in viscous pressure drop during the displacement of the less viscous DNAPL.

Downward displacement of water by TCE is an unstable displacement, with a much more irregular front and several gravity fingers. The interplay between hydrostatic pressure and viscous pressure varies under this condition of displacement and results in an overall decrease of the inlet TCE pressure. As shown in 
Fig. 6b, the measured pressure decreases as the less viscous and denser fluid invades the system due to the constant TCE injection rate. Here, buoyancy forces are destabilizing and dominate the movement, resulting in typical DNAPL fingers in the displaced fluid. The macroscopic water/DNAPL interface is therefore no longer horizontal and many throats become blocked by the capillary effects. The number of active invasion paths is therefore low, and the flow section of DNAPL is reduced. The contribution of viscous pressure is also limited and is dominated by the contribution of the higher density of the invading fluid. Combination of all of these effects results in the decreased inlet pressure.

\subsection{Influence of the DNAPL injection rate on gravity fingering}

The DNAPL injection rate is one of the key factors in displacement of water by DNAPL and controls the balance between the buoyancy, viscous, and capillary forces. Low injection rates yield more opportunities for capillary effects and buoyancy effects to acting in displacement, and the viscous forces are basically limited. It has been previously shown that under a low injection rate, the vertical-downward displacement of water by TCE gives rise to several fingers. Development of fingers is mainly caused by the destabilizing effect of the gravitational forces that dominate the displacement process in this situation. The invasion of a saturated porous medium by TCE is only partial, and the resulting distribution of the pollutant is thus inhomogeneous. To study the effect of increasing the injection rate on the occurrence of instability phenomena during downward flow mode, a displacement experiment was performed with a doubled DNAPL injection rate.

The statistical description of the measured arrival times shows that their distribution is quite similar to the one obtained for upward displacement at a low flow rate (see Fig. 3). The standard deviation is about $17 \mathrm{~s}$, which is significantly less than that obtained for the case of downward displacement of water by TCE at a low rate (standard deviation equal to $70 \mathrm{~s}$ ) and similar to that observed in the case of upward movement (stable case, standard deviation equal to $9 \mathrm{~s}$ ). Thus, the increase in the injection rate significantly stabilizes the displacement. The displacement pattern has more resemblance to the compounding pattern for the vertical-upward displacement recorded at low flow rate. This can be explained by the fact that the high DNAPL injection rate is related to a high-pressure gradient, and when the pressure gradient is high enough, and then both the capillary and buoyancy forces might be effectively neglected. Here $N_{\mathrm{b}}$ is negative $\left(-2.7 \times 10^{-5}\right)$ and the viscosity ratio $M$ is always unfavorable, but an increase of the capillary number $\left(N_{\mathrm{c}}\right.$ increases from $4.1 \times 10^{-6}$ to $\left.7.5 \times 10^{-6}\right)$ seems to lead to a transition to a stable displacement regime which can be referenced in the 3D diagram proposed by Ewing and Berkowitz (Ewing and Berkowitz, 1998). This result is rather surprising, because DNAPL has a lower viscosity, and one would expect a faster injection rate to destabilize the fronts even more. The motion may be unstable, but the shape of the finger head is such that it hits most of the sensors in a moderate amount of time, whereas the untouched parts of the flow are located in the zones without sensors. Alternatively, the separation between a TCE finger and water may cover a longer distance at high speed, which then becomes comparable to or larger than the cell size.

TCE saturations obtained at high injection rate are shown as function of depth in Fig. 5. The TCE saturations are globally higher than those obtained in the case of low injection rate. The average TCE saturation is now increased to $47 \%$. The corresponding profiles also display fewer irregularities than those obtained in the case of downward drainage conducted at a low injection rate. As the flow rate increases, the advancing DNAPL/water front behavior becomes more abrupt.

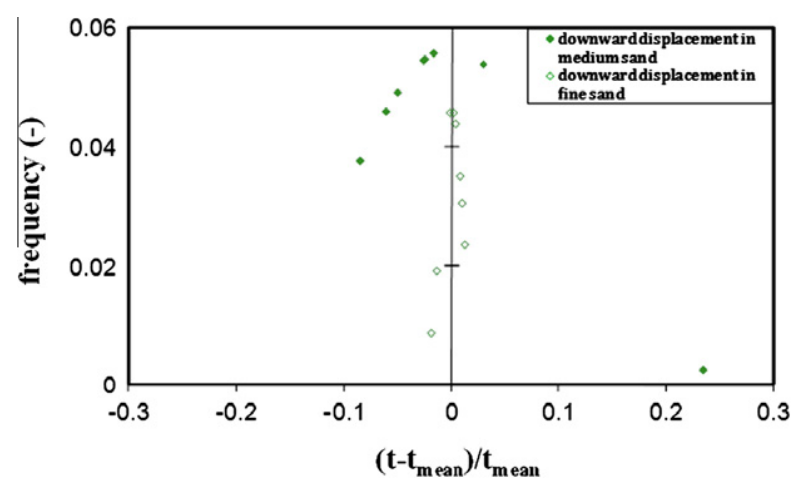

Fig. 7. Measured dimensionless arrival times of the water/DNAPL front in both medium sand and fine sand: case of vertical-downward water displacement at low DNAPL injection flow rate $(40 \mathrm{~mL} / \mathrm{min})$.

\subsection{Effect of permeability on gravity fingering}

To study the effect of the intrinsic permeability of the porous medium on the gravity fingering process, a second displacement experiment was performed using fine sand and applying the same low DNAPL injection rate as in the first experiment. The intrinsic permeability of the fine sand is approximately eighteen times lower than that of the medium sand. Fig. 7 shows the comparison of measured dimensionless arrival times of the TCE front (during the downward displacement of water by TCE) carried out in fine sand to those obtained in the medium sand. In the case of the fine sand, statistical analysis of the determined distribution of front arrival times yields a nearly uniform distribution. The standard deviation in this case is only $12 \mathrm{~s}$, in comparison to the $70 \mathrm{~s}$ recorded in the case of displacement of water by TCE in the medium sand. Because the permeability is primarily controlled by the pore-throat size, changing the size of the mean grain diameter of the sand has a larger effect on drainage displacement behavior due to the increase in capillarity effects (Yao and Hendrickx, 2001). One might expect that the growth of gravity-driven fingers would be nearly suppressed under this condition of displacement. Although buoyancy forces dominate the displacement especially at low DNAPL injection rates and the Bond number is negative, the capillary number $\left(N_{\mathrm{c}}=4.1 \times 10^{-6}\right)$ is closer to the absolute value of Bond number $\left(\left|N_{\mathrm{b}}\right|=4.2 \times 10^{-6}\right)$ in the case of displacement in fine sand than in the case of displacement in medium sand $\left(\left(\left|N_{\mathrm{b}}\right|=2.7 \times 10^{-5}\right)\right.$. Indeed, a lower mean grain size of the porous medium is directly translated to a lower absolute value of the Bond number (see Eq. (3)). This might explain the reduction in the potential contribution of buoyancy forces to the development of fingering processes in low-permeability porous media. Furthermore, when an evolving finger propagates in fine sand, the finger velocity may be slowed down until the non-wetting phase pressure increases enough to overcome the local increase in capillarity effects, which is apparently sufficient to stabilize the displacement front. Indeed, the destabilizing effect of the unfavorable viscosity ratio on the displacement was not completely overcome and formation of local fingers that we did not observe might have occurred.

Based on these observations, we may assert that when the Bond number is negative but have the same magnitude as the capillary number, may corresponding to a stable displacement region that we can refine in the 3D diagram proposed by Ewing and Berkowitz (1998).

\subsection{Residual DNAPL saturations}

Residual TCE saturations were measured in the sand-filled column after a complete drainage-imbibition cycle. Four configuration 
cycles related to the applied flow mode (vertical-upward flow, vertical-downward flow) in both primary drainage and waterflooding processes were carried out in the medium sand. The primary drainage was initially carried out under a stable displacement condition using a constant vertical-upward DNAPL flow rate. This resulted in a high initial TCE saturation, whereas the verticaldownward primary drainage gave rise to a small initial TCE saturation in the saturated porous medium (see Section 3.2). After each drainage experiment, water was then either injected from bottom to top, which reproduced an unstable gravity condition, or from top to bottom in a second experiment, allowing buoyancy forces to play a maximum stabilizing role in the water flooding process. In addition, one complete drainage-imbibition cycle was conducted in the fine sand (see Table 3 ). In this case, both primary drainage and water-flooding displacements were performed in the direction of the gravity forces. Section 3.5 clearly showed that stable conditions were achieved during the drainage process even for a destabilizedgravity condition and unfavorable viscosity ratios between the two fluids.

A large range of water flow rates was applied to mobilize the DNAPL entrapped in the porous medium after primary drainage. Corresponding entrapped TCE saturation in the porous medium was measured by mass balance as described in Section 2.3. The water flow rate was varied (up to approximately four times the initial flow rate) by increasing the water injection rates from 40 to $170 \mathrm{~mL} / \mathrm{min}$, corresponding to a capillary number $N_{\mathrm{c}}$ ranging from $4.1 \times 10^{-6}$ to $16.8 \times 10^{-6}$.

Fig. 8 summarizes the observed average TCE saturations as a function of the capillary number $\left(N_{\mathrm{c}}\right)$ for the four drainage-imbibition cycles conducted in the medium sand and for the one cycle performed in both the fine and medium sand under the same flow mode. It should be noted that, independent of the primary drainage condition (stable or unstable), the average residual TCE saturations quantified in the medium sand were lowest for downward waterflooding and highest for upward water-flooding at the lowest capillary number of $4.1 \times 10^{-6}$. For a high initial TCE saturation in the porous medium (66\%), the remaining TCE saturation is only $18 \%$ for downward water-flooding against $45 \%$ when a upward waterflooding was applied. This result is in good agreement with the findings of Helen and Paul (1997). In the case of upward waterflooding, the viscous forces are counteracted by buoyancy forces at the lowest water-flooding rates, and a large fraction of the TCE remains in the pores. However, buoyancy forces push the DNAPL droplets downward when the water is injected from the top. Whereas the buoyancy forces are cooperative forces in the downward water-flooding and are combined with the capillary and viscous forces to displace the DNAPL droplets, the buoyancy forces are subtractive forces in the case of upward displacement and compete the capillary and viscous forces that mobilize the entrapped DNAPL. Furthermore, the remaining TCE saturations in the medium sand for both flow modes applied during water-flooding tend to decrease as $N_{\mathrm{c}}$ increases. This indicates that both the capillary and buoyancy forces are overcome by the viscous forces at high injection flow rates, and therefore, the mobilization of TCE becomes independent of the chosen flow mode. On the other hand, it can be noted that residual TCE saturations obtained at high capillary numbers were slightly different for both water-flooding directions, whereas their values were fairly equal for the two primary drainage directions and followed the same water-flooding mode. Regardless of the initial saturation in the porous medium, the average residual TCE saturation was stabilized around 20\% and $12 \%$ for upward water-flooding and downward water-flooding, respectively. Therefore, it can be concluded that residual TCE saturations are directly related to the imbibition flow mode and are independent of the initial distribution of DNAPL in the porous medium.
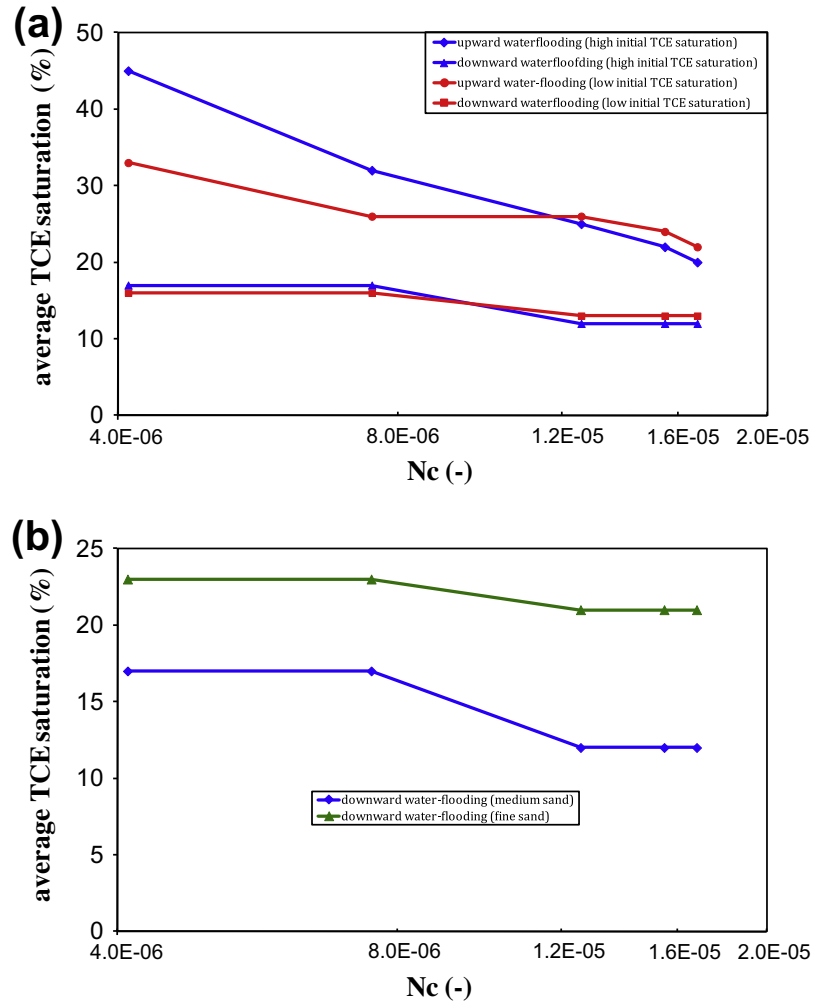

Fig. 8. Average TCE saturations as a function of the capillary number $N_{\mathrm{c}}$ : (a) four drainage-imbibition cycles performed in medium sand and (b) one drainageimbibition cycle performed in medium and fine sand under the same flow modes.

As shown in Fig. 8b, the residual TCE saturation determined at the lowest capillary number $\left(N_{\mathrm{c}}=4.1 \times 10^{-6}\right)$ for the fine sand was slightly higher than that observed in the medium sand. The initial difference of about 5\% increases with increasing capillary number. At higher capillary numbers, residual TCE saturation is reduced to $20 \%$ in the fine sand, and $13 \%$ in the medium sand. This may be explained by the fact that, with decreasing mean grain size of the porous medium, capillary forces become dominant and lead to a significant quantity of TCE being captured in the porous medium, even when viscous forces increase during water-flooding at high flow rates.

Fig. 9a and b shows the measured local TCE saturations at a centre point and two point edges of a column transect as a function of depth for downward water-flooding displacement and upward water-flooding displacement in the medium sand, respectively. In the case of downward water-flooding, the residual TCE saturation seems to be more homogeneous along the whole section of the soil column because the measured TCE saturation values on the axis and edges (two opposite points) of the soil column are closer to each other at most sampling depths than those obtained in the case of upward-water flooding. Despite favorable viscosity ratios existing between the immiscible fluids, fingering driven by destabilizing density may be formed (Lenormand and Zarcone, 1985; Lenormand, 1989). The shape of these fingers might be different to those observed during gravity destabilizing primary drainage. During downward water-flooding, as the displacing fluid (water) is lighter than the displaced fluid (TCE), the TCE/water interface is kept stable, and water most likely crosses the entire section of the sand-filled column. Consequently, the major part of the entrapped TCE in the porous medium is mobilized downwards at the same rate. However, due to the unstable gravity condition in the case of upward water-flooding, water flows upwards along preferential paths and leaves behind a heterogeneous spatial 

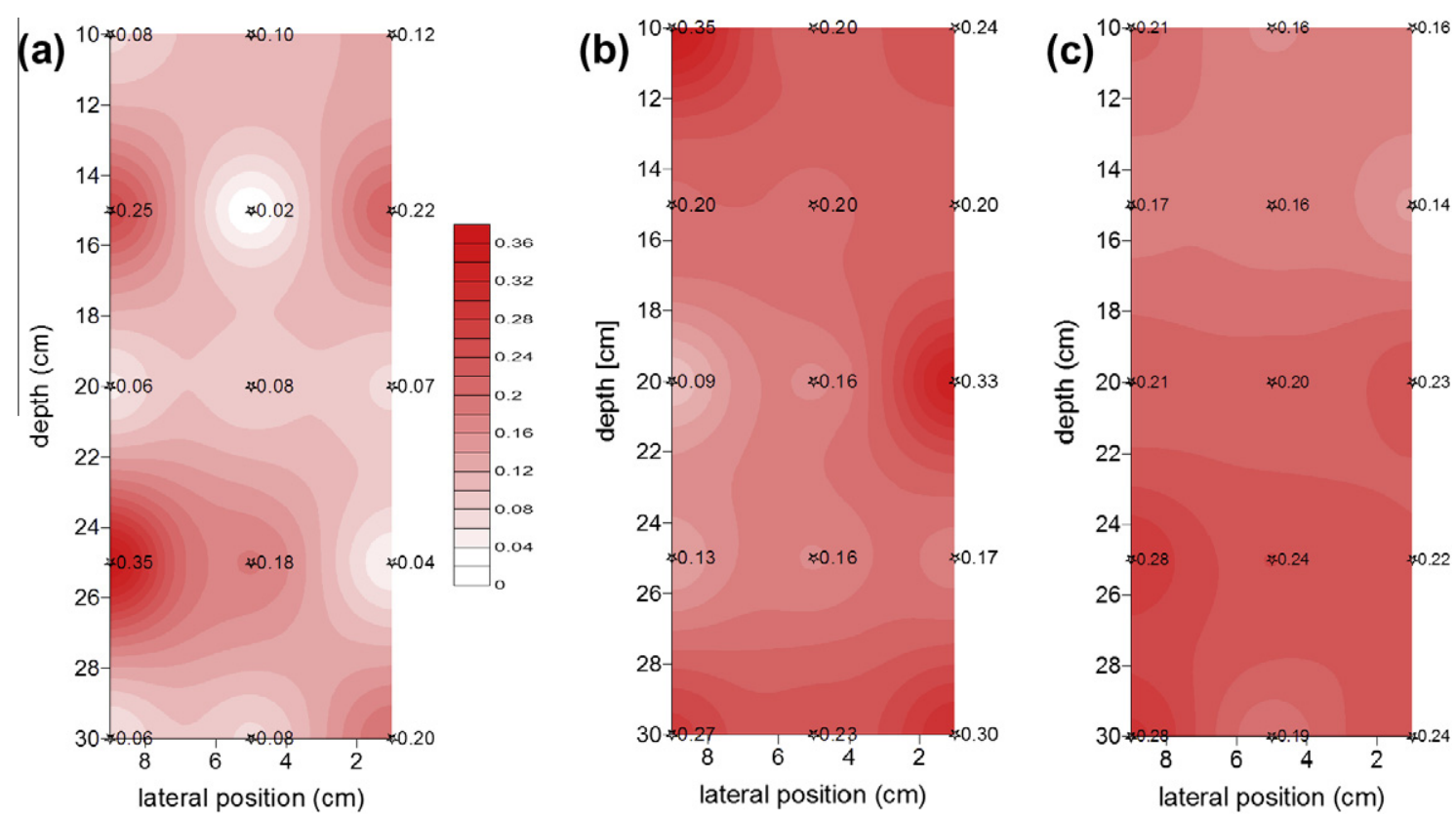

Fig. 9. Residual TCE saturation at different positions of the column section as function of depth: (a) downward water-flooding displacement and (b) upward water-flooding displacement in the medium sand, and (c) downward water-flooding displacement in the fine sand.

distribution of an amount of TCE in the sand. This is shown as an example for a depth of $z=-15 \mathrm{~cm}$ (with $z=0$ corresponding to the top of the column). A low residual TCE saturation of only $2 \%$ was measured, indicating the preferential pathways of water, whereas at another sampling point located at the same depth, a high residual TCE saturation of nearly $25 \%$ was quantified, illustrating a region not in contact with the water flush.

The residual TCE saturation measured in the fine sand as a function of depth at different points of the column section (centre point and two point edges) are shown in Fig. 9c. The residual TCE saturation appears to be more uniformly distributed than in the medium sand. For a given depth, the difference between TCE saturation values in the axis and along the wall of the column is lower than $5 \%$. Furthermore, the average residual TCE saturation varies between $16 \%$ and $24 \%$ along the total sampling height. This indicates that, due to the dominating capillary forces in the fine sand (as previously stated), the immiscible front of displacement is almost abrupt.

\section{Conclusion}

In this study, optical fiber sensors were developed to quantify DNAPL gravity-driven fingering in a water saturated homogeneous porous medium. The influence of several hydrodynamic parameters (such as direction of flow, velocity of displacing fluid, and texture of the porous medium) on the instability displacement process was evaluated during various displacement configurations (drainage experiment and complete drainage-imbibition cycle).

DNAPL front arrival times recorded halfway up in a column filled with medium sand were more spread out in downward drainage than those obtained in the case of upward drainage, showing evidence of preferential path growth for the non-wetting fluid in the saturated porous medium. Here, the spatial distribution of the measured DNAPL saturation was rather inhomogeneous. As expected, buoyancy forces help to stabilize the displacement during the vertical-upward drainage, however, they enhance the unstable displacement and may lead to gravity-driven fingers in the case of a downward displacement. It is worthwhile to note that the velocity of front displacement and the permeability of the porous medium may fundamentally affect the occurrence of gravity fingers and may even entirely suppress the fingering process during immiscible downward displacement. The experimental results highlight that, even for a gravity-destabilized condition, the displacement of the immiscible front was more stable in the case of a high DNAPL injection rate, and entrapped DNAPLs were nearly uniformly distributed in the saturated medium sand. This happens because viscous forces become dominant over buoyancy forces. This stabilizing effect is evident in laboratory experiments, but it is very hard to achieve in a real applied situation. Additionally, it was also concluded from the experimental results that downward migration of the DNAPL in a sand with fine texture leads to an enhanced stabilization of the immiscible front in the saturated porous medium. Here, the small pores of the fine sand accentuate the local capillary pressure in the pores, which slows down the front displacement and makes it more uniform.

The experimentally obtained residual DNAPL saturations clearly indicate that upward water-flooding displacement leads to higher quantities of entrapped DNAPL than during downward displacement of water by DNAPL. It was also found that residual DNAPL saturation is a decreasing function of the capillary number $N_{\mathrm{c}}$, which confirms the findings of earlier studies. Subsequently, the difference in permeability between the fine sand and the medium sand significantly impacts the values of residual DNAPL saturation. Furthermore, the residual DNAPL saturations measured in the case of the fine sand were much more uniformly distributed over the depth than in the medium sand. In an upcoming paper, the experimental data will be used to validate a recently developed numerical grain size distribution based pore-throat model (Nsir and Schäfer, 2010) to compute the water/DNAPL displacement at the pore scale.

\section{Acknowledgements}

Financial support for this research was received from the programme REALISE (REseau Alsace de Laboratoires en Ingénierie et Sciences pour l'Environnement). The Région Alsace, the GDR «Hydrodynamique et Transferts dans les Hydrosystèmes 
Souterrains " (INSU-CNRS), and the Conseil Scientifique de l'Université Louis Pasteur Strasbourg are gratefully acknowledged. Furthermore, the authors would like to thank the anonymous reviewers for their valuable comments and suggestions, which helped to improve the article significantly.

\section{References}

Anderson, M.R., Johnson, R.L., Pankow, J.L., 1992. Dissolution of dense chlorinated solvents into groundwater. 3. Modeling contaminant plumes from fingers and pools of solvent. Environ. Sci. Technol 5, 901-908.

Barczewski, B., Marschall, P., 1992. Development and application of a light fiber fluorimeter for tracer tests. In: Hôtzl, H., Werner, A. (Eds.), Tracer Hydrology. Balkema, Rotterdam, pp. 3-39.

Benremita, H., Schäfer, G., 2003. Quantification du transfert de trichloroéthylène en milieu poreux à partir d'un panache de vapeurs vers la nappe d'eau souterraine. C.R. Méc. 331 (12), 835-842.

Bettahar, B., Ducreux, J., Schäfer, G., Van Dorpe, V., 1999. Surfactant enhanced in situ remediation of LNAPL contaminated aquifers: large scale studies on a controlled experimental site. Transp. Porous Med. 37, 276-286.

Birovljev, A., Furuberg, L., Feder, J., Jøssang, T., Måløy, K.J., Aharony, A., 1991. Gravity invasion percolation in 2 dimensions-experiment and simulation. Phys. Rev. Lett. 5, 584-587.

Bohy, M., Schäfer, G., Razakarisoa, O., 2004. Caractérisation de zones sources de DNAPL à l'aide de traceurs bisolubles: mise en évidence d'une cinétique de partage. C.R. Géosci. 336, 799-806.

Brailovsky, I., Babchin, A., Frankel, M., Sivashinsky, G., 2006. Fingering instability in water-oil displacement. Transp. Porous Med. 63, 363-380.

Christophe, C., Hugues, B., Annie, C., 2010. Drainage in two-dimensional porous media: from capillary fingering to viscous flow. Phys. Rev. E, 82, 046315.

Dicarlo, D.A., 2004. Experimental measurements of saturated overshoot on infiltration. Water Resour. Res, 40, W04215.

Ewing, R.P., Berkowitz, B., 1998. A generalized growth model for simulating initial migration of dense non-aqueous phase liquids. Water Resour. Res. 34 611-622.

Fayers, F.J., Zhou, D., 1996. On the importance of gravity and three-phase flow in gas displacement processes. J. Petrol. Sci. Eng. 15, 321-341.

Ferer, M., Bromhal, G.S., Smith, D.H., 2007. Crossover from capillary fingering to compact invasion for two-phase drainage with stable viscosity ratios. Adv. Water Resour. 30, 284-299.

Frette, O.I., Måløy, K.J., Schmittbuhl, J., Hansen, A., 1997. Immiscible displacement of viscosity-matched fluids in two-dimensional porous media. Phys. Rev. E 3 , 2969-2975.

Geiger, S.L., Durnford, D.S., 2000. Infiltration in homogeneous sands and a mechanistic model of unstable flow. Soil Sci. Soc. Am. J. 64, 460-469.

Gioia, F., Urciuolo, M., 2006. Combined effect of Bond and capillary numbers on hydrocarbon mobility in water saturated porous media. J. Hazard. Mater. 133 218-225.

Glass, R.J., Nicholl, M.J., 1996. Physics of gravity fingering of immiscible fluids within porous media: an overview of current understanding and selected complicating factors. Geoderma 70, 133-163.

Glass, R.J., Conrad, S.H., Peplinski, W., 2000. Gravity destabilized non-wetting phase invasion in macroheterogneous porous media: experimental observations of invasion dynamics and scale analysis. Water Resour. Res. 36, 3121-3137.

Glen, R., Boyd, D., Ana, M., Gómez, O., Minghua, L., Husserl, J., 2006. Effects of initial saturation on properties modification and displacement of tetrachloroethene with aqueous isobutanol. J. Contam. Hydrol. 88, 69-91.

Gupta, B.D., Verma, R.K., 2009. Surface plasmon resonance-based fiber optic sensors: principle, probe designs, and some applications. J. Sensors 15.

Helen, E.D., Paul, V.R., 1997. Influence of viscous, gravitational, and capillary forces on DNAPL saturation. Ground Water 35, 261-269.

Hunt, J.R., Sitar, N., Udell, K.S., 1998. Nonaqueous phase liquid transport and cleanup. I. Analysis of mechanisms. Water Resour. Res. 8, 1247-1258.

Jellali, S., Muntzer, P., Razakarisoa, O., Schäfer, G., 2001. Large scale experiment on transport of trichloroethylene in a controlled aquifer. Transp. Porous Media 44 145-163.
Jellali, S., Benremita, H., Muntzer, P., Razakarisoa, O., Schäfer, G., 2003. A large-scale experiment on mass transfer of trichloroethylene from the unsaturated zone of a sandy aquifer to its interfaces. J. Contam. Hydrol. 60, 31-53.

Khataniar, S., Peters, E.J., 1992. The effect of reservoir heterogeneity on the performance of unstable displacements. SPE Reservoir Eng. 7, 263-281.

Kueper, B.H., Frind, E.O., 1989. An overview of immiscible fingering in porous media. J. Contam. Hydrol. 2, 95-110.

Lenormand, R., 1989. Flow through porous media: limits of fractal patterns. Proc. Roy. Soc. Lond. Ser. A, Math. Phys. Sci. 423, 159-168.

Lenormand, R., Zarcone, C., 1985. Invasion percolation in an etched network: measurement of a fractal dimension. Phys. Rev. Lett. 54, 2226.

Lenormand, R., Zarcone, C., 1989. Capillary fingering: percolation and fractal dimension. J. Porous Media 4, 599-612.

Lenormand, R., Zarcone, C., Sarr, A., 1983. Mechanisms of displacement of one fluid by another fluid in a network of capillary ducts. J. Fluids Mech. 135, 337-353.

Leverett, M.C., 1939. Flow of oil-water mixtures through unconsolidated sands. Trans. Am. Inst. Min. Metall. Pet. Eng 132, 149-171.

Løvoll, G., Méheust, Y., Toussaint, R., Schmittbuhl, J., Måløy, K.J., 2004. Growth activity during fingering in a porous Hele Shaw cell. Phys. Rev. E 70, 026301.

Løvoll, G., Méheust, Y., Måløy, K.J., Aker, E., Schmittbuhl, J., 2005. Competition of gravity, capillary and viscous forces during drainage in a two-dimensional porous medium, a pore scale study. Energy 30, 861-872.

Løvoll, G., Jankov, M., Måløy, K.J., Toussaint, R., Schmittbuhl, J., Schäfer, G., Méheust, M., 2011. Influence of viscous fingering on dynamic saturation-pressure curves in porous media. Transp. Porous Med. 86 (1), 305-324, doi:10.1007/s11242 010-9622-8.

Méheust, M., Løvoll, G., Måløy, K.J., Schmittbuhl, J., 2002. Interface scaling in a twodimensional porous medium under combined viscous, gravity and capillary effects. Phys. Rev. E, 66, 051603.

Nguyen, V.H. Sheppard, A.P., Knackstedt, A.A., Pinczewski, W.V., 2006. The effect of displacement rate on imbibition relative permeability and residual saturation. J. Petrol. Sci. Eng. 52, 54-70.

Nicholl, M.J., Glass, R.J., 2005. Experimental observations of gravity-driven fingering during immiscible displacements within a rough-walled fracture. Vadose Zone J. 4, 1123-1151.

Nsir, K., 2009. Etude expérimentale et numérique de la migration des polluants non miscibles à l'échelle de Darcy. PhD thesis, Université de Strasbourg, France, 207.

Nsir, K., Schäfer, G., 2010. A pore-throat-model based on grain size distribution to quantify gravity-dominated DNAPL instabilities in water saturated homogeneous porous medium. C.R. Geosci. 342, 881-891.

Parsons, R.W., Marathon, O.C., 1975. Microwave attenuation - a new tool for monitoring saturations in laboratory flooding experiments. SPE J. 15, 302-310.

Pennell, K., Pope, G., Abriola, L., 1996. Influence of viscous and buoyancy forces on the mobilization of residual tetrachloroethylene during surfactant flushing. Environ. Sci. Technol 30, 1328-1335.

Riaz, A., Tchelepi, H.A., 2006. Influence of relative permeability on the stability characteristics of immiscible flow in porous media. Transp. Porous Med. 64 315-338.

Saffman, P.G., Taylor, G., 1958. The penetration of a fluid into a porous medium or Hele-Shaw cell containing a more viscous liquid. Proc. Roy. Soc. Lond., Ser. A $245,312-329$

Schwille, F., 1988. Dense Chlorinated Solvent in Porous and Fractured Media: Model Experiments. Translated from the German by Pankow. J.F. Lewis Publishers, Boca Raton, Florida, pp. 1-146.

Sharma, A.K., Gupta1, B.D., 2006. Fibre-optic sensor based on surface plasmon resonance with Ag-Au alloy nanoparticle films. Nanotechnology 17, 124-131.

Theodoropoulou, M.A., Sygouni, V., Karoutsos, V., Tsakiroglou, C.D., 2005. Relative permeability and capillary pressure functions of porous media as related to the displacement growth pattern. Int. J. Multiphase Flow 31, 1155-1180.

Toussaint, R., Løvoll, G., Méheust, Y., Måløy, K.J., Schmittbuhl, J., 2005. Influence of pore-scale disorder on viscous fingering during drainage. Europhys. Lett. 71 583-589.

Tullis, B.P., Wright, .S.J., 2007. Wetting front instabilities: a three-dimensional experimental investigation. Transport Porous Medium 70, 335-353.

Yao, T., Hendrickx, J.M., 2001. Stability analysis of the unsaturated water flow equation. 2. Experimental verification. Water Resour. Res. 7, 1875-1881.

Zhang, Z.F., Smith, J.E., 2001. The velocity of DNAPL fingering in water-saturated porous media: laboratory experiments and a mobile-immobile-zone model. J. Contam. Hydrol. 49, 335-353. 